\section{ACUTE INFLAMMATION OF THE AORTA ASSOCIATED WITH PROFOUND MYOCARDIAL DISEASE.}

\author{
BY F. J. POYNTON, M.D. LOND.,
}

CASUALTY PHYSICLAN TO ST. MaRY'S HOSPITAL.

CASES are recorded from time to time in which, with or without angina, death occurs quite suddenly and in which the necropsy reveals a condition of inflammation at the base of the aorta unusually acute in its character and remarkably localised in its distribution. In these cases the aortic valves may be thickened by chronic inflammation and yet they may nevertheless be quite competent, and neither during life nor after death can aortic regurgitation be demonstrated and brought forward as an explanation of the sudden cardiac failure. The area of the aorta which is so gravely and severely diseased is the one from which the coronary vessels arise, and it is a natural suggestion that the heart wall has suffered in its nutrition either from a narrowing of the lumen of one or both of these arteries, or that the ressels have shared in the same acute process, and their ramifications have become occluded as a result. A reference to the literature upon this subject proves that such may be the case in some instances, but in the two cases which are recorded below I do not think that such an explanation is tenable. In neither of these two cases were the orifices of the coronary vessels certainly diminished in size nor were their walls visibly diseased, and microscopic examination of the heart wall showed that their ramifications were not occluded. In both cases the cardiac wall was examined with especial care and in both the changes found were widespread and severe and of a nature analogous to that found in the aorta. It is difficult to escape from the conviction that there had been a common antecedent cause which had thus injured these two organs which anatomically and physiologically are so closely associated. The changes in the aorta were obtrusive and in the first of the recorded cases those in the heart wall might macroscopically have been easily overlooked, yet in both cases I should suppose the sudden death depended far more upon these less obtrusive signs in the heart than they did upon the striking changes in the vessel wall. The question of the etiology of this condition is one of great difficulty and it will perhaps be the most rational course to record the two cases with the necropsies and microscopy before allusion is made to their causation. I am indebted to Dr. Oheadle for the first case, under whose care the patient had been while in St. Mary's Hospital.

CASE 1.-A married woman, aged 38 years, was admitted to St. Mary's Hospital under the care of Dr. Cheadle on Feb. 8th, 1899, suffering from pain in the region of the heart and vomiting. Two months before Christmas she had first noticed occasional attacks of pracordial pain extending down the left arm which she said became blue and useless. These attacks, infrequent at first, had during the last week become frequent and severe and were accompanied by vomiting and faintness. Except for a severe attack of "influenza" four years before, from which she completely recovered, her health had been good. She had one healthy child and there had not been any miscarriages. She was said to have been addicted to alcoholism and to have been maltreated by her husband. Her father was still alive, aged 78 years, and her mother died in childbirth. The patient was well nourished and on admission was quite comfortable and free from pain, but shortly after reaching the ward she had a slight attack of pain in the chest and left arm with a feeling of faintness. Dr. Oheadle saw her during this attack and considered it to be one of true angina. The next day she was quite free from pain and she then volunteered the statement that any sudden exertion, even sitting up in bed, was liable to bring on the pain. The temperature was normal. The pulse was of low tension with a small wave, it varied in rate considerably, but was about 72 when the clinical notes were taken. At times it was excited and irregular. The cardiac impulse was in the fifth space internal to the nipple line and the area of dulness was slightly increased to the left. The first sound at the apex was sharp and was preceded occasionally by a short presystolic murmur. The second sound was reduplicated both at the apex and the base. The second sound at the base was sharp and there was no suspicion of a diastolic murmur. The urine was natural, of specific gravity 1025 , and there was no evidence of disease in the other organs. The whole of this day and the following were passed in comfort, but at midnight on the 11th a severe anginal attack commenced. The pain radiated over the præcordial region, between the shoulders and down the left arm, and was accompanied by severe vomiting. The face became deathly pale and the extremities cold and clammy. The pulse was rapid and irregular and the first sound reduplicated over the whole præcordial area. The patient was in great distress and complained of vertigo and loss of vision. This condition, in spite of every attempt at relief, continued with remissions and exacerbations until $3 \mathrm{~A} . \mathrm{M}$. when the patient died. For the account of the anginal attack and the major part of the clinical notes I am indebted to Dr. Sumner, house physician, and to Dr. Cheadle.

The necropsy was made 32 hours after death. The pericardium was natural and the heart itself was slightly dilated The cavities were empty and the large veins were gorged with blood. There was a white patch upon the right ventricle and the cardiac muscle was pale and pink in colour. The aortic valves were distinctly thickened but quite competent. The pulmonary valves were natural. The mitral valve was affected by a chronic valvulitis which had slightly narrowed the orifice. The posterior flap had been most affected and the chordæ tendineæ in one part were so shortened that at this spot the valve was tethered on to the papillary muscle. In the substance of the valve there was a calcareous nodule The tricuspid orifice and valve were natural. Neither ventricle was hypertrophied. The left auricle was slightly thickened. The commencement of the aorta for the first two inches was extensively diseased and this condition extended in irregular patches along the arch. The intima of the vessel in this situation was raised from the general surface and was pinkish red in colour, where the disease was most acute. Some of these raised patches had a translucent appearance; others were yellow with a red flush around their bases. A few patches resembled the ordinary condition of atheroma. The appearance was that of an acute aortitis supervening upon a more chronic inflammation. The large vessels springing from the aorta were not affected. The orifice of the right coronary artery appeared narrower than usual, but it admitted a large probe easily, and the walls of both vessels were macroscopically healthy. The other large arteries were not diseased. There were peritoneal adhesions the result of a previous inflammation between the left lobe of the liver and the spleen; and in the midst of these adhesions there was a calcareous nodule of the size of a large marble. Except for this peritonitis there was no evidence in the other viscera of lesions which might have been explained by syphilis. The bones were only examined by palpation, but no results of a localised chronic inflammation were detected in any of them. The kidneys macroscopically and microscopically were healthy.

Microscopy of the aorta and cardiac muscle,-The sections of the aorta were made from tissue fixed in corrosive sublimate and embedded in paraffin. The intima was swollen and the vessels were distended. Around the vessels there was a free exudation of cells and the fibrons tissues were swollen. In places the cell exudate reached the surface of the intima. The elastic coat was also freely infiltrated with leucocytes which extended to some depth into its substance. Where the aorta merged into the heartwall itself the exudation could be traced beneath the endocardium of the left ventricle.

The cardiac wall.-Both the left and the right ventricle were examined, the former in three different places and the latter in two. Some of the material was fixed in sublimate and some in Hermann's fluid. All the sections were cut in paraffin. Extensive changes were found in both ventricles which, speaking generally, consisted of scattered foci of advanced disease surrounded by areas comparatively unaffected. The morbid changes are considered under the following headings: (1) the nuclear changes; (2) the changes in the muscle protoplasm; (3) the changes in the interstitial tissues; and (4) the changes in the vessels.

1. Changes in the muclei.--The nuclei stained with carbolthionin took the dye but feebly as compared with the muscle protoplasm, which was coloured a deep blue, whereas in 
healthy muscle the nuclei stain a deep violet and the protoplasm a faint blue. This condition I have noticed in other cases of sudden death-for example, in a case of gastric alcer in a young woman in which the necropsy disclosed no definite explanation, in a case of dilated heart of obscure origin, and in a case of acute septicxmia. Sections fixed in Hermann's fluid and specially stained with nuclear stains showed the nuclei to be in many fibres almost colourless, and if a decolourising method was used they rapidly lost the stain instead of retaining it tenaciously. 'This condition was most obvious in the neighbourhood and within the foci of marked disease. Stained with methyl green the nuclei also showed this feeble staining reaction.

2. The muscle protoplasm had in many places completely lost striation; in other parts this was still evident. In addition there was marked longitudinal striation, a fraying of the ends of the muscles, and in some places frequent transverse fracture. Where the disease was advanced many dark granules were obvious around the nuclei and in some fibres this change was so extensive that only a shell of muscle fibre and a faint nucleus could be seen. In other parts the muscle had a homogeneous hyaline appearance. The osmic acid preparations showed fatty changes in some fibres and in those which were most diseased this was extensive. If treated with very dilute acid the yellow granules and black fat globules were apparent in the same fibre.

3. The interstitial tissue showed some increase, but this was not striking. Where disease was most advanced the increase in the interstitial elements was more apparent than real, being due to the complete destruction of the muscular fibres, leaving the connective tissue framework more than usually apparent. In some places there was a cellular infiltration between the muscle fibres and an appearance of active reaction.

4. The large vessels showed in some of the sections a considerable increase in the thickness of the outer coat, but there was not an endarteritis. No occluded vessels were detected in a number of sections which were examined especially for them. The capillaries in some of the diseased areas were distended with blood and were much more obvious than usual. The disease was irregularly distributed in all parts of the ventricle with no particular relation to the pericardium though some of the foci reached the surface. No organisms were detected in the aorta or the cardiac wall.

CASE 2.-A man, aged 63 years, was brought to St. Mary's Hospital on Nov. 10th, 1898. No account could be obtained of his previous health or habits. The circumstances of his death were the following. He was sitting at a table in a shop at 2.30 P.M. shortly after dinner when while speaking to his fellow-workmen he suddenly got up from the table and. fell to the ground. He was then found to be unconscious and lay in this condition, breathing heavily for 10 minutes. He was placed in a cab but died before reaching the hospital.

The post-mortem examination was made 24 hours after death. Rigor mortis was present; there was much abdominal and a considerable amount of epicardial fat. The heart weighed 18 ounces. Both ventricles were dilated and the left was slightly hypertrophied. The endocardium in the left ventricle was opaque and thickened. The aortic valve was competent, and the segments were distinctly thickened. The mitral flaps were thickened; the other valves were natural. The cardiac muscle, especially in the interventricular septum and toward the apex of the left ventricle, was obviously fibrosed. The commencement of the aorta showed an extensive inflammation of the inner coat. It was red and showed also raised translucent patches and yellow areas, the latter older in date and less acute in their origin than the former. The orifices of the coronary arteries were natural in size and there was no visible disease in their walls. The kidneys microscopically showed some fibrosis and the vessels were distended with blood. There were no evidences of visceral syphilis in the other organs.

Microscopy of the corta.-The intima was increased in thickness and the vessels were distended. There was extensire cellular exudation, both reaching the inner surface of the ressel and extending into the elastic coat

The curdiac muscle was extensively diseased in scattered areas. Some of the fibres showed striation, but many had lost all vestige and there was some fatty change and many granules around the nuclei of these fibres. The interstitial changes were very extreme in some parts of the interventricular septum. The muscle had almost completely disappeared and the outer coats of the vessels were thickened. The smaller vessels in places were distended and there was exudation of leucocytes, pointing to an active process.

There is a striking similarity between these two cases and one which would in all probability have been even greater had any previous history of the second case been obtainable. Both died rapidly but not abruptly and in both acute changes were found at the commencement of the aorta, with marked focal changes in the cardiac walls, and these latter in the absence of definite disease of the coronary vessels. In neither case was the pericardium affected: In both there was some thickening of the mitral and aortic valves, but in neither were the aortic valves incompetent. In the man the heart was considerably enlarged, but it must be borne in mind that he was 63 years of age and that his kidneys showed some cirrhosis. That the etiology of such cases as these is obscure can be readily understood when there is so little that is definite to be gathered from their history. The condition of the heart and aorta goes far to explain the symptoms but helps but little to explain the causation.

The microscopic evidences of dilated vessels, exudation of leucocytes, and increase of fibrous tissues point to a reaction to some morbid process, and the activity of it is manifested in the destruction of the muscular fibres and altered condition of the intima of the artery. Morbid processes of many different kinds may, however, produce a like reaction to that presented in these cases and the remarkable feature lies not so much in this as in the peculiar distribution of the disease. In the case of the woman I examined all the larger arteries with especial care and saw no trace of a condition similar to that found in the aorta. The literature upon the subject is to some extent unsatisfactory, for the condition of aortitis is in many instances considered apart from that of myocarditis and vice versâ. In some cases the changes in the cardiac wall are undoubtedly due to disease of the coronary vessels, while of a case recorded by Dr. G. W. Balfour (an illustration of which forms a frontispiece to his "Clinical Lectures on Diseases of the Heart and Aorta") it is stated that the heart was almost healthy, though there were some narrowing of the coronaries and interference with the circulation. French authors have written with great care and minuteness upon the subject of aortitis, both clinically and experimentally, and it is well recognised that such a condition may occur as secondary to an acute infection, as in typhoid fever, variola, or influenza, or may apparently be primary. In these two cases it is not, I think, a question of a primary or secondary aortitis which has to be considered but a joint affection of the heart and artery dependent upon some common cause. A combination such as this was observed by Landouzy and Scredery in hæmorrhagic small-pox and typhoid fever, though the inflammation was more acute and in the arteries far more extensive. In infective endocarditis the aorta and cardiac wall may be both extensively diseased. Boinet and Romany have produced aortitis in animals by injecting diphtheria toxins under the skin and Mollet and Regaud and other observers have produced myocardial disease by the same methods. There'is then evidence of this combination occurring as a result of infections, the disease in the heart and arteries not being dependent the one upon the other but both attributes of the particular infection.

In these cases there was not any evidence of an acute infection and the appearance of the vessels pointed to a condition of acute disease supervening upon a more chronic process, which would appear to be the rule in the case of acute primary aortitis. One would therefore naturally turn for the explanation to some chronic toxæmia in which there are exacerbations of intensity, and syphilis is perhaps the most readily brought to mind. This disease is known to affect the arteries, but especially the smaller ones, and also the myocardium. In 1856 Sir (then Dr.) Samuel Wilks described extensive fibroid changes in the heart as a result of syphilis, and in 1897 Dr. Sidney Phillips ${ }^{1}$ published an important paper upon the same subject. Unfortunately, there is no account of the aorta except incidentally, the paper dealing solely with the changes in the heart itself. In the case of the woman recorded in this article the chronic inflammation between the left lobe of the liver and spleen with the calcareous nodule enclosed therein is very suggestive of such a cause, but further evidence there was none. The condition of the cardiac wall in both cases would be compatible with syphilitic disease, but, as already stated, in the absence of a definite gummatous change it 
seems unwise to dogmatise. Alcoholism is another possibility, for there was a fairly definite history of the habit in the first case, and the appearances at the necropsy in the second were quite compatible with such a theory. The effect of this poison upon the cardiac muscle is known to be very profound and its influence in producing inflammation in the larger vessels is also undoubted.

The immense importance of the clinical aspect of these cases is evident and the diagnosis and prognosis of the greatest practical interest. In this article the pathological aspect has been especially dwelt upon, for the other aspects are dealt with as fully as it is possible by various writers upon diseases of the heart, Dr. G. A. Gibson, ${ }^{2}$ in his work upon the heart, for example, devoting considerable attention to those points which bear upon diagnosis and symptomatology. The first case exemplifies the difficulty and doubt which surround theit recognition. It is the pathological association of disease in the aorta and heart muscle itself apart from gross affection of the coronaries to which I have here called especial attention, for it is a point which has not been as clearly stated in the writings npon this subject as I think it deserves.

Queen Anne-street, W.

\section{A CASE OF EPITHELIOMA OF THE TONGUE: SURVIVAL FOR OVER 23 YEARS AFTER REMOVAL OF THE GROWTH.}

Br CHRISTOPHER HEATH, F.R.C.S. ENG SEXIOR SURGEON TO UNIVERSITY COLLFGE HOSPITAL.

$A^{\prime}$ the present time when attempts are made to prove that cases of malignant disease which survive an operation for three years may be regarded as "cures" I wish to put on record a remarkable case of survival in perfect health for over 23 years. I extract the following report of the case from the Transactions of the Pathological Society of 1876.

"Epithelioma of the tongue involving the loner jaw; removal.-A man, aged 52 years, was admitted into University College Hospital under Mr. Heath's care in September, 1875, with very extensive cancerous disease of the tongue and sublingual structures. In January, 1874, was noticed a swelling of one of the submaxillary glands and soon after a sore beneath the tongue. The sore healed and the gland subsided under treatment. In September, 1874, the gland began to swell and at last broke. At the same time he found that he had difficulty in articulating as the tongue was fixed to the floor of the mouth and eventually the tip of the tongue became fixed to the jaw. This was temporarily relieved in April, 1875, by an operation undertaken in a London hospital. On admission the patient was unable to protrude his tongue or move it in his mouth. The saliva constantly trickled away and articulation was very imperfect. He complained of great pain in the occipital region but of none in the tongue. The gums of the incisor region were swollen and ulcerated and the teeth were loose. The tongue was fixed to the back of the jaw which was softened. All the tissues beneath the jaw were indurated, but the skin was not involved. On Sept. 29th, 1875, Mr. Heath removed the tongue, the centre of the jaw, and all the sublingual tissues by dividing the skin in the middle line, then sawing the jaw through on each side, and, having isolated the tongue somewhat on each side, by enclosing the whole of the disease with the wire of the galvanic écraseur. The patient made a good recovery and was shown to the society on Nov. 2nd. The parts removed consisted of the middle three inches of the lower jaw, nearly the whole of the tongue, and the sublingual muscles and glands en masse. At the posterior end the mass measured two and a half inches in depth and slightly more from side to side. The tong ue appeared to be healthy except at the anterior part; and on the left side, just behind the tip, was a nodule of the size of a pea. Beneath the tongue was a mass of yellowishwhite firm tissue with a granular surface which was continued quite up to the cut margin. This tissue on microscopical examination proved to be epithelioma.

Examined microscopically, the gums and the tissues

2 Diseases of the Heart and Aorta, 1898. at the root of the tongue, even to the edge of the part, are found to be epitheliomatous. Characteristic 'globes' are only very small and few in number. but collections of large epithelial cells of varied shapes, with clear distinct nuclei, are very abundant and characteristic. The growth is infiltrating the muscles at the root of the tongue, but the tongue itself except at the tip is free.'

The patient completely lost the occipital pain of which he complained and which I have noticed in other cases of cancer of the tongue but cannot explain. The two halves of the jaw gradually fell together and firmly united, as 1 have seen in more than one similar case, and the stump of the tongue grew considerably. Articulation and mastication were very fair and the patient presented himself periodically before my class until two years ago when, getting too old to work, he went into the country where he died in January, 1899.

In the Transactions of the Pathological Society for 1898 Mr. Littlewood records a case of sarcoma of the tongue and refers to one which I reported in the same Transactions in 1869. The case was then recorded as ono of "medullary cancer of the tongue," but an elaborate report upon it by the Committee on Morbid Growths is given which shows it to have been really a sarcoma-a growth at that date not differentiated. The specimen consisted of the anterior half of the tongue, which had been removed with the écraseur after section of the lower jaw for a mass involving the organ and the floor of the mouth. The patient was 60 years of age and the disease had existed six months, and on looking into his mouth there was between the tongue and the lower jaw on the left side a ragged ulcerated surface occupying the floor of the mouth. The operation was performed in September, 1869, and the patient lived till 1884, when be died from senile decay, aged 78 years.

Cavendish-square, w.

\section{ON THE TREATMENT OF INSANITY BY VARIOUS ANIMAL EXTRACTS.'}

BY ARTHUR W. WILCOX, M.B., C.M. EDIN.

SEXIOR ASSISTANT MEDICAL OFFICER, WARWICK COUNIY ICYATIC ASYLUM.

1 WISH to call attention in this paper to some brief records of cases of different forms of insanity treated by varions glandular extracts. The extracts used were orarian, cere brinin, didymin, and thyroid_all in the form of tabloids, each containing five grains. In every case the usual precautions to be followed before beginning a course of gland-feeding were taken-viz, apparently sound bodily condition on careful physical examination of the patient and rest in bed for a few days before and after as well as during treatment. except in one case where the dose was small and continued for a longer period than usual. During the time when the patients were confined to bed their diet consisted chiefly of milk, beef-tea, light puddings, \&c. The dose given at the outset was 15 grains and it was increased daily by 15 grains until 60 grains had been given. The treatment was usually continued for five, six, or seven days, but longer if thought advisable.

The cases chosen for ovarian feeding were those in which there was complete amenorrhoea, the most successful results being obtained in the insanity of adolescence. Female ep:leptics were treated by cerebrinin, male epileptics by didymin, while thyroid was administered in cases of melarcholia, acute mania, and dementia. Five patients were subjected to ovarian extract treatment, the use of this tabloid being suggested to me under circumstances whicb. are explained in Case 1

CASE 1.-The patient, who was admitted to the Warwick County Lunatic Asylum in November, 1897, suffering frors: mania, was a fairly nourished girl, aged 19 years, and was stated to have had a previous attack which was treated at home. She was delusional, inclined to be violent, very restless, and occasionally faulty in her habits. About a montin after admission she became more demented, wandered abou. in an aimless manner, smiled fatuously when addressed, and grew very dirty and depraved in her habits. This dementec

A paper read before the Northern and Midland Branch of the Medis: Psycholugical $\Lambda$ ssociation ou April 12th, 1859 . 